\title{
Physician Perspectives on Severe Behavior and Restraint Use in a Hospital Setting for Patients with Autism Spectrum Disorder
}

\author{
Giovanna L. Salvatore $^{1} \cdot$ Christina A. Simmons $^{1}$ (I) $\cdot$ Patrice D. Tremoulet $^{1}$ \\ Accepted: 7 October 2021 / Published online: 16 October 2021 \\ (c) The Author(s), under exclusive licence to Springer Science+Business Media, LLC, part of Springer Nature 2021
}

\begin{abstract}
Hospitals, with many features that can evoke severe behavior in patients with autism spectrum disorder (ASD), often use restraint as a behavior management strategy. Prior research on restraint in patients with ASD has primarily focused on children or specific departments. Twenty-five physicians and medical trainees from an urban teaching hospital participated in discussions about experiences managing severe behavior in patients with ASD across the lifespan. Twenty themes emerged from thematic analysis of participant transcripts. The five most salient themes included: lack of procedural knowledge with restraint implemented by other hospital professionals; alternative strategies to manage severe behavior; negative perceptions of restraint; helpful role of caregivers; and limited experience treating patients with ASD, and critical need for training in function-based management.
\end{abstract}

Keywords Restraint $\cdot$ Autism spectrum disorder $\cdot$ Severe behavior $\cdot$ Hospital

Severe behavior, such as aggression, self-injury, and property destruction, is more common in individuals with ASD than their neurotypical peers (Newcomb \& Hagopian, 2018). The majority of research on severe behavior in individuals with ASD is limited to children, and prevalence estimates vary widely. Hill et al. (2014) estimate that between 8 and $68 \%$ of children with ASD engage in aggressive and destructive behavior, while others estimate that as many as $82 \%$ (Murphy et al., 2009) to $93.7 \%$ (McTiernan et al., 2011) exhibit challenging behavior. This discrepancy in estimates has been attributed to differing operational definitions of severe behavior, assessments used, and study participants (Hill et al., 2014).

Severe behavior may cause property damage or lead to injury of self or others (Kanne \& Mazurek, 2011; Newcomb \& Hogopian, 2018); it can impair social relationships with

Christina A. Simmons

simmonsc@ rowan.edu

Giovanna L. Salvatore

salvat38@students.rowan.edu

Patrice D. Tremoulet

tremoulet@rowan.edu

1 Department of Psychology, Rowan University, 201 Mullica Hill Road, Robinson Hall Suite 117, Glassboro, NJ 08028, USA peers, family and community members; and it may also lead to social stigmatization (Werner \& Shulman, 2013). Individuals with ASD who engage in severe behavior likely require more costly healthcare, education, residential, habilitative, and vocational services than individuals with ASD without severe behavior (Buescher et al., 2014; Hill et al., 2014). In addition, severe behavior may interfere with skill acquisition and on-task behavior, which may ultimately affect educational and vocational status and attainment (DeavenportSaman et al., 2016; Emerson et al., 2001).

Applying restraint is a common strategy for managing severe behavior, despite associated increases in an individual's risk of depression, anxiety, and post-traumatic stress disorder following restraint implementation (Evans \& Cotter, 2008; Friedman \& Crabb, 2018). Mechanical restraint, which entails using equipment such as arm splints or waist straps to restrict movement, may result in skin breakdown and difficulties with balance, strength, and gait, loss of muscle mass, and infection (Evans \& Cotter, 2008). Use of physical restraint, in which at least one person uses their body to restrict an individual's ability to move their torso, arms, legs or head, is associated with distrust of medical professionals (Wong et al., 2020). Both physical restraint and chemical restraint, which entails using medication (e.g., Benzodiazepines) to inhibit patient movement and manage emergent behaviors, can cause serious injury, functional decline, and 
even death (Friedman \& Crabb, 2018). Restraint can be implemented both reactively to manage emergent behaviors and proactively to facilitate medical compliance and access to medical care (Allen et al., 2009; Kupzyk \& Allen, 2019).

Initiatives to reduce restrictive behavior management for individuals with ASD, such as staff training, reformed organizational policies, and mindfulness-based interventions, are associated with positive outcomes, including improved safety and decreased long-term costs (Sturmey, 2018). Nonetheless, restraint is still used in institutional, residential, day habilitation, vocational, and school settings. Similarly, although healthcare professionals have identified alternative approaches to restraint (e.g., clear communication, visual cues; Johnson \& Rodriquez, 2013), and researchers have suggested that physicians should only use restraint after all safer alternatives have been exhausted (Blumberg $\&$ Roppolo, 2021), many hospitals currently use restraint to manage behaviors in patients with a variety of diagnoses (Schnitzer et al., 2020).

The decision to implement restraint in healthcare settings is multifactorial in that patient characteristics, including diagnoses; care team's knowledge, experience and attitudes; organizational policies and culture; and physical environment all interact during split-second decisions about whether or not to restrain a patient (Roy et al, 2019). One hospital system witnessed a drastic culture shift in how its medical providers viewed and used restraint after instituting a program that included more expansive training and education, goal-setting across departments, multidisciplinary rounds, and alternative equipment (e.g., soft belts, arm sleeves; Cosper et al., 2015). Although many healthcare providers recognize that restraint should be a last resort, researchers have reported an increased risk of inappropriate restraint use in children with ASD and intellectual disability due to limited staff training and knowledge of ASD (Gabriels et al., 2012). More recent research indicates that children with a diagnosis of ASD and intellectual disability continue to experience higher rates of restraint compared to those without those diagnoses (O’Donoghue et al., 2020).

For adult patients, alcohol or drug use and psychiatric conditions increase the likelihood of restraint. Age, ethnicity, and gender also influence healthcare providers' decisions about using restraint (Grimes, 2012; Mann-Poll et al., 2011). One study reported that a majority of restrained patients were perceived as a danger to self or others (60.6\%) or non-compliant or unwilling to follow directions $(28.1 \%$; Wong et al., 2019). Although these studies present valuable information on predictors of restraint, little research specifically focuses on use of restraint for adult patients with ASD, whose acute medical care needs might differ from those of other adult patients.

Moreover, many healthcare providers have characterized their knowledge and practical skills in treating patients with
ASD as poor or fair, though mental health providers tend to have more knowledge and skills than providers of adult medicine and obstetric/gynecological services (Zerbo et al., 2015). Even neurologists have reported less comfort treating adult patients with ASD than adults with other neurological disorders (Oskoui \& Wolfson, 2012). Similarly, pediatric and family physicians have rated their competency treating children with ASD as lower than their competency treating children with other neurodevelopmental disorders (Golnik et al., 2009).

Deficits in knowledge and lower confidence about treating patients with ASD is concerning as it may affect physicians' ability to deliver safe and effective treatment to patients with ASD. In particular, physicians may over-rely upon restraint, when function-based treatments are known to be more effective (Campbell, 2003; Heyvaert et al., 2014). These treatments are developed by identifying the maintaining consequence (i.e., function) of the patient's behavior, such as access to attention, escape from aversive tasks or stimuli, and sensory stimulation (Iwata et al., 1982/1994), access to a tangible item (Day et al., 1988), and routine restoration (Hagopian et al., 2007). For example, a patient with ASD may engage in severe behavior to escape the distress caused by bright lights in an exam room. For this patient, dimming the lights could be a simpler, safer, more effective strategy than restraint. Kupzyk and Allen (2019) reviewed behavioral interventions to increase medical compliance, finding that graduated exposure and contingent reinforcement are most commonly used followed by modeling and prompting, distraction or relaxation, and behavioral momentum. However, numerous barriers to implementing function-based treatment may exist, including a lack of resources, time, provider knowledge, and interdisciplinary collaboration, and concern with insurance reimbursement (Mazurek et al., 2020).

As the prevalence of ASD increases (Christensen et al., 2019), healthcare providers are likely to treat more patients with ASD. Moreover, individuals with ASD use hospital services more frequently than others. Adolescents with ASD alone are four times more likely to visit emergency rooms annually than their neurotypical peers (Liu et al., 2017), and individuals with ASD frequently have comorbid medical and psychiatric concerns (e.g., anxiety, depression, and gastrointestinal symptoms) which may necessitate emergency medical services (Lunsky et al., 2014; van Steensel et al., 2011). Further, Lunsky et al. (2014) reported that children with ASD commonly engage in severe behavior in hospitals, even if their presenting concern at the time of hospitalization was medical. For instance, one patient engaged in severe head banging due to frustration and another engaged in aggression due to an undetected urinary tract infection causing pain at the time of the medical evaluation (Lunsky et al., 2014).

Meanwhile, hospital settings can be particularly challenging for individuals with ASD who experience social 
communication challenges, sensory sensitivity, and routine rigidity (American Psychiatric Association, 2013). A patient in a hospital setting may encounter unfamiliar physicians, bright lights, loud noises, crowded spaces, and unpredictable routines, any of which could evoke severe behavior (Hazen et al., 2020; Muskat et al., 2015). In addition, frustration surrounding communication with healthcare providers may contribute to the occurrence of severe behavior. Communication challenges experienced by patients with ASD may also contribute to physician difficulty understanding and interpreting pain, physical discomfort, or signs and symptoms of medical conditions in patients with ASD (BroderFingert et al., 2016). Thus, presenting medical concerns alone may not predict the level of care an individual with ASD and comorbid severe behavior requires during their hospital stay.

As a result, some families have developed autism-specific care plans (ACPs) to improve experiences of healthcare for patients with ASD and their families. These ACPs may include communication preferences and strategies, environmental modifications, and safety concerns. Initial research indicates that ACPs could help to improve the experience of pediatric patients with ASD and their families; however, use of ACPs for treating patients with ASD across the lifespan and their efficacy across various hospital departments and diverse gender and racial groups has not been established (Broder-Fingert et al., 2016).

In general, little is known about physician experiences managing severe behavior in patients with ASD. Similarly, most of the established literature on restraint occurrence and predictive variables is focused on those without ASD or pediatric patients with ASD. Taken together, the increasing incidence of ASD in the United States, higher rate of hospitalization experienced by individuals with ASD, features of hospitals that could trigger severe behavior in individuals with ASD, and serious implications of restraint compared to alternative behavior management strategies indicate a critical need for healthcare workers to be prepared to use methods other than restraint to manage severe behavior in patients with ASD. An initial step toward meeting this need is exploring physicians' experiences in managing severe behavior in hospital patients with ASD.

\section{Method}

Multidisciplinary focus groups and interviews were conducted with medical trainees and early-career physicians, respectively, who work in a large urban teaching hospital in the Northeastern United States; such hospitals tend to experience the highest rates of patients with ASD (Lokhandwala et al., 2012). Discussions focused upon the following topics: (a) experiences with severe behavior management in patients with ASD, including restraint implementation; (b) treatment differences across patients and departments; (c) relevant training received; (d) relevant knowledge of ASD and behavioral function; and (e) perceived needs. This research complied with the American Psychological Association Code of Ethics and was approved by Rowan University's Institutional Review Board. Informed consent was obtained from each participant.

\section{Recruitment}

A total of 22 medical trainees (students, $n=17$; residents, $n=4$ ) and three early-career physicians were recruited from an urban hospital and affiliated medical school via direct emails from the research team, emails from hospital leadership and hospital administrators (e.g., Dean, Student Affairs Officer, program director, department chairs or heads), and posted recruitment flyers. Medical trainees included students, residents, and fellows who were currently in or had recently completed a rotation in the target departments of Emergency Medicine, Psychiatry, Pediatrics, and Neurology. Early career physicians (herein referred to as "physicians") were serving in their first 1-to-5 years of post-supervised practice in one of the target departments. Departments most likely to treat patients with ASD were selected for inclusion.

\section{Participants}

A majority of participants were female (68\%) and medical students (72\%). Races included White (52\%), Asian (20\%), Hispanic (16\%), and African American (12\%). All target departments were represented, with many medical trainees selecting multiple departments to reflect their rotations across disciplines. Primary departments included: Pediatrics (80\%), Neurology (76\%), Psychiatry (76\%), Emergency Medicine (72\%), Surgery (76\%), Internal Medicine (76\%), Family Medicine (72\%), and Obstetrics/Gynecology (64\%). Table 1 presents participant demographics.

\section{Focus Groups with Medical Trainees}

Each focus group included six to eight participants, as guided by previous health research (Bender \& Ewbank, 1994). Focus group participants were assigned to their respective group based on overlapping availability. Two trained facilitators co-conducted three virtual focus groups using Cisco WebEx ${ }^{\circledR}$ v.40.11.4.15, a HIPAA-compliant video conferencing platform. The facilitators were located in separate private areas where discussions could not be overheard. Facilitators requested that participants join from a private and confidential area and that they remain unmuted with video cameras on during the entire session. The chat feature was disabled to more closely resemble an in-person 
Table 1 Demographics

\begin{tabular}{|c|c|c|}
\hline Variable & $N$ & $\%$ \\
\hline \multicolumn{3}{|l|}{ Gender } \\
\hline Female & 17 & 68 \\
\hline Male & 8 & 32 \\
\hline \multicolumn{3}{|l|}{ Race } \\
\hline White & 13 & 52 \\
\hline Asian & 5 & 20 \\
\hline Hispanic & 4 & 16 \\
\hline African American & 3 & 12 \\
\hline \multicolumn{3}{|l|}{ Status } \\
\hline Student & 18 & 72 \\
\hline Resident & 4 & 16 \\
\hline Physician & 3 & 12 \\
\hline \multicolumn{3}{|l|}{ Department } \\
\hline Pediatrics* & 20 & 80 \\
\hline Neurology* & 19 & 76 \\
\hline Psychiatry* & 19 & 76 \\
\hline Emergency medicine* & 18 & 72 \\
\hline Surgery & 19 & 76 \\
\hline Internal medicine & 19 & 76 \\
\hline Family medicine & 18 & 72 \\
\hline $\mathrm{OB} / \mathrm{GYN}$ & 16 & 64 \\
\hline Pediatric emergency & 4 & 16 \\
\hline Anesthesia & 2 & 8 \\
\hline Student clinic & 2 & 8 \\
\hline NICU & 2 & 8 \\
\hline Integrative medicine & 1 & 4 \\
\hline PICU & 1 & 4 \\
\hline Radiology & 1 & 4 \\
\hline Ultrasound & 1 & 4 \\
\hline Urology & 1 & 4 \\
\hline
\end{tabular}

Department $=$ all current departments/rotations completed. Asterisk indicates departments targeted during recruitment. Participants could endorse multiple departments to represent current placement or rotations completed

$O B / G Y N$ Obstetrics/Gynecology, NICU neonatal intensive care unit, $P I C U$ pediatric intensive care unit

interaction and to promote active participation. Each focus group was audio and video recorded.

At the start of each session, facilitators requested that participants only discuss experiences treating patients at the hospital from which they were recruited. Any examples from other institutions were redirected and were omitted from data analysis. The facilitators used a semi-structured interview guide with open-ended questions about participants' perceived needs with regard to treating patients with ASD, their training, and their experiences managing and documenting severe behavior and restraint. See Appendix 1 for the question guide. When the lead facilitator judged that the session had reached a point of saturation, and no new information or themes were being discussed, she ended the session.

One medical trainee had to leave the first focus group early due to an unexpected emergency. All other focus group members actively participated for the entire duration of their sessions, which may be attributed to the online format and enhanced perception of anonymity (Stewart \& Shamdasani, 2017) or the relevance of topics to all participants (Malterud et al., 2016). Comments from the trainee who left early were included in the qualitative data analysis.

\section{Interviews with Physicians}

Due to significant challenges scheduling physicians for a focus group session, three physicians were interviewed instead, using the same structured interview guide.

\section{Autism Stigma and Knowledge Questionnaire}

After focus groups and interviews were ended, participants (except the trainee who had to leave her focus group early) were directed to complete an online version of the Autism Stigma and Knowledge Questionnaire (ASK-Q; Harrison et al., 2017). The ASK-Q measures participants' perceived knowledge of the core features of ASD, and yields a total knowledge score and the following subscale scores: (a) diagnosis, (b) etiology, (c) treatment, and (d) stigma. The ASK-Q was selected due to its strong psychometric properties, including high internal consistency (Cronbach's Alpha $=0.88)$ and test-retest reliability across subscales (range 0.93 to 0.98 ; Harrison et al., 2017). Cross-cultural utility of the ASK-Q was indicated by adequate internal consistency (Cronbach's Alpha $=0.72$ ) and high test-retest reliability (ICC $=0.86$; Harrison et al, 2019). This questionnaire took a median of $3.5 \mathrm{~min}$ to complete (range 2.4 to $18.1 \mathrm{~min})$.

\section{Data Preparation}

Recordings of the focus groups and interviews were transcribed for analysis. Participants were described by participant number with no identifiable information. Transcripts were thematically analyzed using the constant comparative method of qualitative data analysis (CCM; Glaser \& Strauss, 1967; Strauss, 1987), as the study's goal included identifying physician experiences and perceived needs, rather than provisional hypothesis testing.

Researchers coded transcript data into explicit categories to establish theory and highlight salient themes. They followed the six phases of thematic analysis in psychology as described by Braun and Clarke (2006): (1) becoming familiar with the data, (2) identifying initial codes of interesting ideas, (3) searching for themes, (4) reviewing themes, 
(5) defining themes, and (6) generating a scholarly report. Data were sorted into themes by comparing text to previous entries or introducing new themes. As themes emerged, operational definitions were created and revised. Subthemes were used to accurately reflect theme content and facilitate consistent coding across the two coders. Data that could have been coded in multiple themes were standardized by establishing specific rules and exclusionary criteria to promote interobserver agreement by independent data analysts. Interobserver agreement, based upon randomly selecting and double coding $33 \%$ of each focus group and interview transcript was $91.7 \%$. The majority of disagreements were omission $(77.5 \%)$, where one coder missed a theme, and the rest were commission, where coders disagreed in theme assignment.

To quantify salience within and across participants, the total occurrences of each theme was summed for each participant and summed across participants (Morgan, 1997). Each example or rationale provided by participants was counted as one occurrence. A separate occurrence was documented once the participant provided a different rationale or another participant responded. Revisiting a previous example or rationale was counted as a separate occurrence. Participant salience for each theme was determined by dividing the frequency of occurrences in one theme by the total occurrences in all themes.

\section{Results}

Mean duration of focus groups was 115.7 min (range 109 to $119 \mathrm{~min}$ ), and mean duration of interviews was $39 \mathrm{~min}$ (range 36 to $41 \mathrm{~min}$ ). A total of 20 themes were identified across all discussions. Table 2 shows frequency and percentage of themes by participant status. The mean rank order correlation between rankings for each focus group and the overall focus group rankings was 0.69 (range 0.46 to 0.86 ), representing a strong positive correlation. In the first focus group, all 20 themes emerged. During remaining focus groups, 19 of the 20 themes were represented. The five most salient themes, in order of salience at the focus group level included:

1. Trainees are not responsible for managing severe behavior and implementing restraint, and were thus unfamiliar with comprehensive restraint protocols.

2. Trainees described or suggested alternative strategies used by themselves or others for treating patients with ASD and severe behavior.

3. Trainees discussed negative reactions or perceptions of restraint by themselves, physicians, caregivers, and patients.
Table 2 Frequency and percentage of themes by participant status

\begin{tabular}{|c|c|c|c|c|c|}
\hline \multirow[t]{2}{*}{ Theme } & \multirow[t]{2}{*}{ Description } & \multicolumn{2}{|c|}{ Medical trainees } & \multicolumn{2}{|c|}{ Physicians } \\
\hline & & $\#$ & $\%$ & \# & $\%$ \\
\hline 1 & Deferred responsibility/limited restraint protocol & 60 & 12.40 & 19 & 10.22 \\
\hline 2 & Alternative strategies severe behavior & 48 & 9.92 & 17 & 9.14 \\
\hline 3 & Negative perception restraint & 40 & 8.26 & 22 & 11.83 \\
\hline 4 & Caregivers helpful & 36 & 7.44 & 16 & 8.60 \\
\hline 5 & Limited ASD experience & 29 & 5.99 & 4 & 2.15 \\
\hline 6 & Internal causes severe behavior & 28 & 5.79 & 6 & 3.23 \\
\hline 7 & Lack of ASD training & 28 & 5.79 & 9 & 4.84 \\
\hline 8 & Observable predictors restraint & 27 & 5.58 & 23 & 12.37 \\
\hline 9 & External predictors restraint & 25 & 5.17 & 6 & 3.23 \\
\hline 10 & Limitations/improvements service delivery & 23 & 4.75 & 10 & 5.38 \\
\hline 11 & Negative description severe behavior & 19 & 3.93 & 5 & 2.69 \\
\hline 12 & Lack crisis training/knowledge & 17 & 3.51 & 8 & 4.30 \\
\hline 13 & Higher tolerance ASD & 14 & 2.89 & 4 & 2.15 \\
\hline 14 & Restraint algorithms & 14 & 2.89 & 7 & 3.76 \\
\hline 15 & Documentation limitations & 13 & 2.69 & 13 & 6.99 \\
\hline 16 & External causes severe behavior & 13 & 2.69 & 4 & 2.15 \\
\hline 17 & Different severe behavior types & 13 & 2.69 & 2 & 1.08 \\
\hline 18 & Proactive medication use & 13 & 2.69 & 2 & 1.08 \\
\hline 19 & Negative perception ASD & 12 & 2.48 & 3 & 1.61 \\
\hline 20 & ASD knowledge & 12 & 2.48 & 6 & 3.23 \\
\hline
\end{tabular}

Themes with equal frequency were ranked in order of highest percentages of each focus group \# number of occurrences; $\%$ percent of total occurrences 
4. Trainees reported the helpful role of accompanying caregivers during patient appointments in the treatment of patients with ASD and severe behavior.

5. Trainees indicated limited practical experience treating patients with ASD and that experiences may vary by hospital department.

\section{Theme 1: Restraint Implementation and Protocols}

Medical trainees reported that other hospital professionals were responsible for implementing restraint, so they were not consistently able to report restraint protocols. Sub-themes included: (a) consulting others (i.e., nurses, rapid response teams, security, psychiatry, child life specialists, behavioral medicine) to help manage severe behavior; (b) removing themselves from the situation, (c) observing protocols implemented by others (e.g., physicians ordering restraint); and (d) noting that protocol knowledge and responsibility may vary by status (e.g., resident vs. medical student) and department (e.g., increased responsibility of psychiatry in restraint protocols). For example, one participant noted, “... we have a procedure called ... behavior rapid response team ... for when a patient's behavior is escalated ... and I'm not sure how often it's used with patients with autism." Another described management of severe behavior as, "having the security staff nearby and nurses nearby and technicians who are very brave people and can be there ready to assist" and a third indicated, "the nurses ... are usually the ones to suggest [restraint] and then I believe that the physicians have to put in the order for it."

\section{Theme 2: Alternative Strategies to Treat Patients with ASD and Severe Behavior}

When asked to describe their response to patients with ASD engaging in severe behavior, medical trainees reported alternative strategies to restraint that they have used personally or have observed used by others, and also proposed strategies they would use. Subthemes included: (a) adapting the physical environment (e.g., moving patient to a different physical location, reducing noise and distraction, reducing number of professionals present during appointment); (b) changing their medical approach (e.g., calmer/quieter tone, altering or forgoing medical assessments, conducting a more comprehensive physical examination to avoid undetected medical issues, attempting to involve the patient, providing simple explanations and repetition); (c) using alternative equipment, if available (e.g., helmet, pillow); (d) providing access to attention or tangible items (e.g., active play, access to stethoscope) and reduced demands (e.g., allowing the patient to engage in special interests and/or repetitive behaviors that may otherwise interfere with medical examinations, breaks); and (e) encouraging appropriate behavior and discussing reinforcers with the patient. One participant commented, "I also try to be really careful to keep things like as low stimulation as possible, so bringing my voice down, not being any louder than necessary." Others stated, "I think [it] was handled very well by the resident, because they did all the things that [the parent] said, made sure that the patient got a room away from a lot of the noise", and "... when the physician would talk to the patient, they'd kind of talk about what are things that the child with autism would want to do after the appointment." Another shared, "I had a little girl and she was on the autism spectrum and she was really fixated on my stethoscope, so while I was examining her, I just let her just play with my stethoscope and it just made things a lot more [sic] easier."

\section{Theme 3: Negative Reactions and Perceptions of Restraint Implementation}

Medical trainees shared negative reactions/assessments of their own and of physicians, caregivers, and patients. Subthemes included: (a) observing patient expressions of pain or resistance to restraint, (b) observing patient disinterest in further medical care post-restraint, (c) observing caregivers having sad or ambivalent reactions to restraint, (d) avoiding reporting restraint use to caregivers, (e) having the desire to remove restraint, (f) perceiving restraint as restricting patient's rights or treating a patient as nonhuman, (g) perceiving the use of restraint as a last resort, and (h) reporting observations of restraint implementation as uncomfortable. One trainee reported, "I think her grandmother was with us and her reaction was both like she was very sad, but at the same time she knew it was the right thing." Others stated, "So now we had to move him and treat him like an animal"; "I just remember, like the scared intern watching patients get put down and it was like, horrifying"; and "the last thing we want to do is put someone down into four-point restraint against their will."

\section{Theme 4: Helpful Role of Caregivers During Appointments for Patients with ASD}

Focus group participants indicated that accompanying caregivers were often helpful in managing severe behavior. Sub-themes included caregivers: (a) providing a model of how to best interact with the patient for the medical trainee to follow, (b) assisting with communication and triggers to severe behavior, (c) implementing restraint rather than other hospital professionals, (d) distracting patients, and (e) providing a sense of comfort. One participant stated, “... when I'm dealing with pediatric patients in particular, I tend to follow suit with the parent or the caregiver, because they 
know more about that child than I do ... they understand their communication a little bit better, and so I try to mimic or shadow what mom is doing, or dad is doing to kind of leverage the child to participate in the exam or interview." Another shared, "Instead of using the papoose, we let the mom hold, which we kind of typically don't do, just because it's like a lot easier to just papoose them, you can place the sutures or remove them. But instead, you know, having the mom kind of be the restraint there so that we could remove the staples."

\section{Theme 5: Limited Practical ASD Experience and Experience Varying by Department}

Medical trainees indicated that experience may vary by hospital department, and thus, departments that serve more individuals with ASD (e.g., neurology and pediatrics) are perceived to have more successful patient interactions and treatment of patients with ASD. They also noted that treatment of unrelated concerns in patients with ASD does not build self-perceived competency specific to treating patients with ASD. Participant comments included, "I've probably only taken care of a handful of patients in ED with autism" and "[In] other departments ... neurology, or wherever there may be other comorbidities where you see autism ... interactions were handled better."

The remaining 15 themes are grouped by categories for ease of discussion.

\section{Category A: Training, Knowledge, and Treatment Specific to ASD (Themes 7, 19, 20)}

Focus group participants indicated that medical school training included only a few lectures and/or didactics about the core features of ASD; they identified formal training in ASD as a gap in their curriculum. They reported that most of their knowledge about ASD was gained through direct patient experiences, from their mentors, or pursuing knowledge themselves, such as from the American Psychological Association (Theme 7). One participant noted, "I wish that there was more formal training, but there's not, at least not, there wasn't for me," while another explained "[treating patients with ASD is] something I've been taught to do by my faculty, but again nothing formal." A third commented, "I've really never received any training or formal education on the treatment of autism in terms of like the disease progression. And I think that that's like a huge miss now that we're talking about it, and honestly, I hadn't reflected about it."

Although participants had limited experience with patients with ASD and severe behavior, they exhibited knowledge of the core features of ASD (Theme 20). Their comments indicated an awareness of: (a) sensory sensitivities, (b) communication differences, (c) fixations/restricted interests, and (d) differences in severity of ASD. Responses also revealed negative perceptions about treating patients with ASD among some medical professionals, such as viewing patients with ASD as more challenging or difficult to treat (Theme 19). For example, "I don't know if it's my own bias that I'm like, 'Oh, this person or child has autism, I must treat them differently.' And so I feel like that unconscious or subconscious bias comes in a little bit."

\section{Category B: Severe Behavior in Patients with ASD (Themes 6, 11, 13, 16, 17)}

Participants reported higher tolerance for severe behavior in patients with ASD, noting that their response for patients with an ASD diagnosis who are engaging in severe behavior may be different compared to their response to others without an ASD diagnosis (Theme 13). They suggested that patients with ASD may exhibit severe behavior due to internal causes (Theme 6) such as: an inability to communicate, loss of control, pain, a coping mechanism and/ or self-regulation, anger or feeling misunderstood, sensory stimulation/overload, and a low frustration tolerance, but also commented that severe behavior might be caused by external factors (Theme 16) such as the hospital environment being loud, scary, and/or intimidating; severe behavior having been reinforced previously; and an inappropriate or failed intervention.

Participants shared negative perceptions and/or evaluations of severe behavior in medical settings (Theme 11), and their comments indicated that they differentiate between different types of severe behavior (Theme 17). Behaviors likely to be considered severe are those perceived as violent, aggressive, threatening, harmful to oneself or others. For example, "Anytime I think of severe behavior, I would say it's anything that poses an immediate threat to the individual themselves or to anybody around them." Meanwhile repetitive behaviors, especially some self-injurious behaviors where a single instance is unlikely to cause immediate harm (e.g., skin picking) and disproportionate reactions were considered "challenging" rather than severe. One participant distinguished crisis behavior from severe behavior as follows, "I think the word crisis has a time element to it versus severe behavior could just be sort of like a descriptive term, or like a noun with an adjective sort of added to it, that doesn't have to be as time sensitive."

\section{Category C: Restraint of Hospital Patients (Themes $8,9,14,18$ )}

Participants described specific strategies for deciding whether restraint was warranted (Theme 14). They also described different responses to severe behavior, depending on its type. In particular, reported restraint was implemented 
faster for self-injurious behavior than for aggression, and faster for physical aggression than for verbal aggression. Another factor influencing decision-making included whether hospital staff safety or patient safety was at risk. Participants reported using a step-wise progression, such as first verbal de-escalation, then chemical restraint, followed by physical restraint. A participant explained, "But if it was just like aggressive, like verbal behavior ... then I would certainly like not necessarily jump to physical or chemical restraint if I could try to deescalate the situation in other ways. But if I were in, you know, harm's way, then I would move towards more physical restraint or ... chemical restraint."

Medical trainees also noted that, in their experience, observable patient characteristics (Theme 8) such as age, race, gender and size, behavioral factors such as suicidality, homicidality or non-compliance or threatening behavior, and non-observable factors (Theme 9) such as criminal status, diagnosis, communication ability/language barrier, cognitive ability may all influence decision-making about restraint implementation. They also indicated that geographic location, time of day, and accessibility of alternatives in a given department can influence restraint decisions. One trainee noted, " ... if it was a patient coming off the street and especially like being in [city], there are so many different stereotypes about like drug seeking behaviors and things like that, that kind of jump to other conclusions." Related to age, another participant stated, "But certainly, I'd be more inclined to restrain someone older because I would think that they're - it could escalate to a point that's more dangerous than a-like child." Focus group participants indicated that age is associated with perceived threat such that older participants who engage in severe behavior may be perceived as more threatening than their younger counterparts. Participants reported that age is often compounded by additional factors (e.g., size, height, weight) which may increase the physician's perception of threat, and thus, lead to a higher incidence of restraint for older patients with ASD. They also noted differences in available resources for managing severe behavior across the lifespan. For example, a child may be accompanied by a caregiver who may offer additional sources of assistance such as managing severe behavior themselves or providing a high-preferred tangible item (e.g., iPad, toy) to compete with a potentially aversive situation. Medical trainees also reported specialized teams that are available for enhanced care of younger patients (e.g., Child Life Specialists), whereas those resources or additional sources of support may not exist for adult patients with ASD.

Finally, participants reported medication was sometimes used as a proactive strategy for treating patients with ASD (Theme 18), including prescribing medication for medical procedures, and administering medication during the appointment to limit behaviors perceived to interfere with the medical care. For example, "I think because of his known behavior, and, you know, not wanting to risk, you know, him biting or getting worked up, and for his comfort, we opted to do like a full sedation for the procedure."

\section{Category D: Limitations to Treatment of Patients with ASD and Severe Behavior (Themes 10, 12, 15)}

Noting that institutional policies impact the treatment of patients with ASD who engage in severe behavior, participants identified a need for unified protocols to manage severe behavior in hospitals. They also indicated that lack of training in working with patients with ASD who may exhibit severe behavior and limitations in existing documentation systems negatively impact treatment of these patients (Theme 10). Medical trainees shared ideas for training, such as, “... a formal training on what even is severe behavior, how is that defined?"; "a simulated patient situation with patients with autism"; "having like a protocol ... whether it's something that can be included within [electronic medical record system], or if it's posted up, just because I think we like algorithms to follow"; and "training ... [to be] able to communicate effectively, both with patients and families when trying to provide medical care."

Participants also reported that inconsistent documentation of severe behavior and current diagnoses in the electronic health record system inhibit physicians' ability to effectively treat individuals with ASD (Theme 15). In particular, they cited inconsistent use of pop-ups and/ or flags to alert them that the patient may exhibit severe behavior, electronic records lacking comprehensive patient information, limited documentation accessibility across external healthcare systems, and current diagnoses not accurately reflected in the presenting concerns section of the patient electronic health record. For example, one participant stated, "It's not like a specific red flag that says like the patient has been agitated before, it's more of just like something that you might figure out through digging, at least in my experience," and another explained, "Even for like a restraint note itself, you would have to pretty much just kind of flip through all the different notes to see if there happens to be one for a behavioral rapid response or something like that."

Finally, participants expressed a lack of foundational knowledge, training, and experience regarding crisis (Theme 12). They added that crisis knowledge and training vary by department and/or specialty. Comments included, "I got absolutely [no crisis training] in medical school, even though I think that really would have been important. And then even in residency training, I feel like it's limited." 


\section{Interviews with Physicians}

During each of the three physician interviews, all 20 themes identified during medical trainee focus groups were discussed. Four of the five most salient themes from the medical trainee participants (i.e., restraint implementation and protocols, alternative strategies, negative perceptions of restraint, role of caregivers) were also in the five most salient themes for the physician participants. The eighth most salient theme for medical trainees "observable patient characteristics are predictor of restraint" was the most salient theme in the physician interviews. The fifth most salient theme for medical trainees "limited ASD experience" moved down to sixth in salience in the physician interviews. The mean rank order correlation between each interview and the overall interview ranking was 0.69 (range 0.55 to 0.85 ) and the mean rank order correlation between focus groups and interviews was 0.66 . See Table 2 for frequency and percentage of themes by participant status.

\section{Autism Stigma and Knowledge Questionnaire}

Medical trainees demonstrated a mean score of 15.2 out of $18(S D=1.4)$ in the diagnosis/symptoms subscale, 12.4 out of 16 ( $S D=2.2$; range 12 to 18 ) in the etiology subscale, 12.3 out of $14(S D=1.4$; range 7 to 15$)$ in the treatment subscale, 0.9 out of 7 ( $S D=1.0$; range 0 to 3$)$ in the stigma subscale, and 39.9 out of $48(S D=3.8$; range 30 to 45$)$ in the total score. All trainees demonstrated adequate scores in the diagnosis/symptoms subscale, $80.9 \%$ in the etiology subscale, $90.4 \%$ in the treatment subscale, $90.4 \%$ in the stigma subscale, and $95.5 \%$ in the total score.

Physicians obtained a mean score of 15 out of $18(S D=2$; range 13 to 17 ) in the diagnosis/symptoms subscale, 14.7 out of $16(S D=0.6$; range 14 to 15$)$ in the etiology subscale, 13.3 out of $14(S D=1.2$; range 12 to 14$)$ in the treatment subscale, 0.3 out of 7 ( $S D=0.6$; range 0 to 1 ) in the stigma subscale, and 43 out of $48(S D=1$; range 42 to 44$)$ in the total score. Physicians demonstrated $100 \%$ adequate total and subscale scores. See Fig. 1 for individual scores and means across the medical trainee focus groups and physician interviews.

\section{Discussion}

Aims of this exploratory research were to (a) gather qualitative information about how physicians manage severe behavior in patients with ASD and (b) identify variables that may affect physician decision-making in restraint implementation. Existing restraint research has largely focused on general patient populations (e.g., Grimes, 2012; Larue et al., 2009) or examined restraint use within a single department (e.g., psychiatry or emergency; Delaney \& Fogg, 2005; Wong et al., 2019, 2020). A few studies have considered neurodiverse populations or specifically individuals with ASD, but
Fig. 1 ASK-Q results. $F G$ focus group, Solid line: maximum score, Dashed line: adequate score

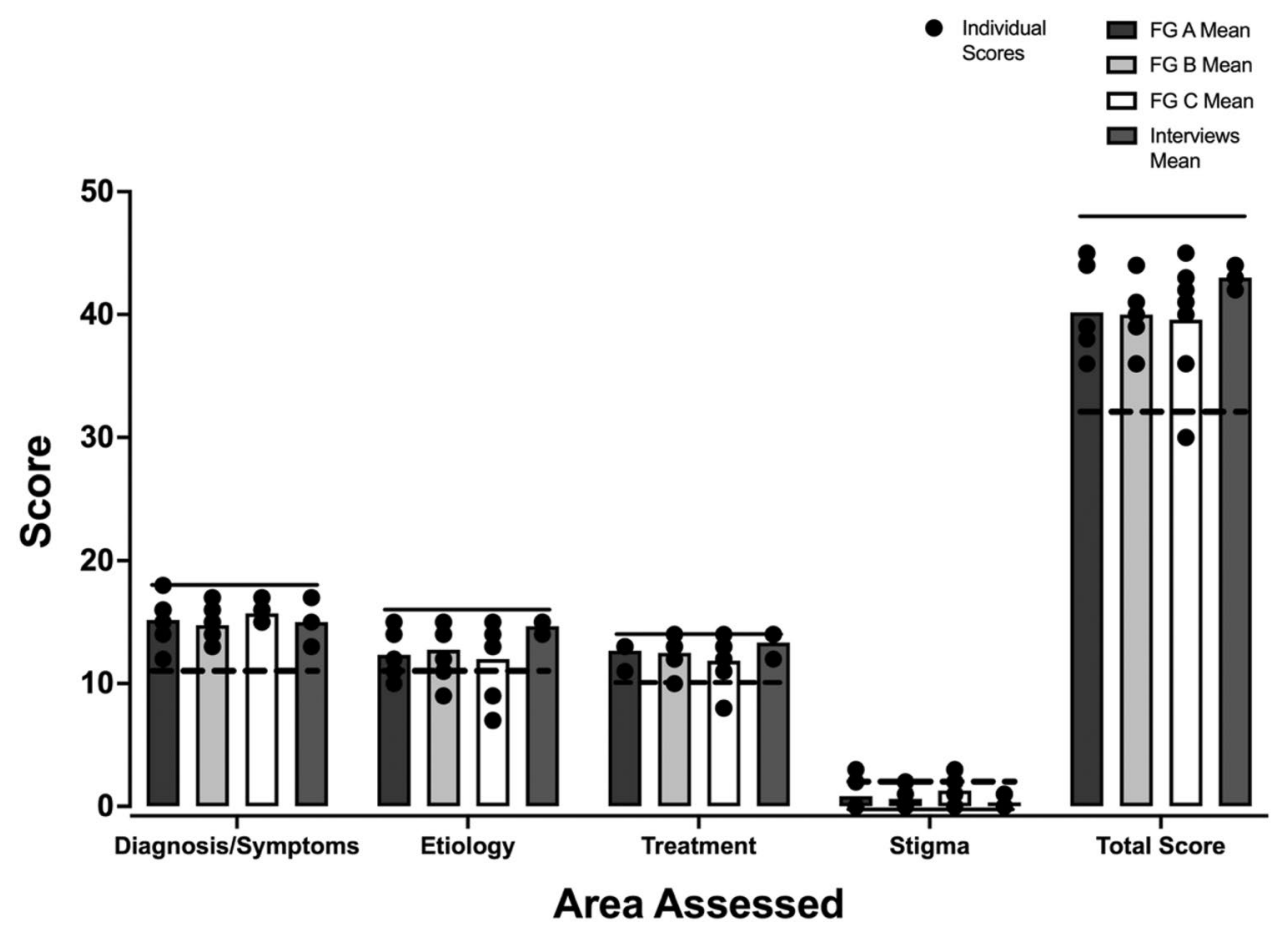


those investigations were limited to pediatric populations (Friedman \& Crabb, 2018; Johnson \& Rodriquez, 2013; O'Donoghue et al., 2020). The current study focused on hospital treatment of patients with ASD and severe behavior across the lifespan, including input from medical trainees and physicians in multiple hospital departments. Although all study participants were affiliated with the same facility, the wide array of patients served in the targeted departments of this urban level 1 trauma teaching hospital resemble patients seen in other hospital systems across the nation. The majority of participants identified as White, which is similar to national physician demographics; however, there was a higher representation of women and racial/ethnic minorities in our sample compared to national estimates provided by the Association of American Medical Colleges (2019).

This study did not find differences between medical trainees' and physicians' experiences with and perspectives about severe behavior in patients with ASD. Both groups articulated strong need for enhanced education about treating patients with ASD and more training in severe behavior management, both during medical school and early career practice. Both groups also identified a need for institutional policies that can help improve service delivery for patients with ASD. Importantly, even though the behavioral literature has widely examined behavioral interventions for increasing compliance with medical procedures (e.g., Kupzyk \& Allen, 2019; Riley \& Freeman, 2019), neither the medical trainees nor the physicians in this study demonstrated a strong understanding of behavioral function, as evidenced by attributing severe behavior to internal factors such as "maintaining inner peace."

Interestingly, participants reported that they generally consulted other hospital professionals to manage severe behavior, assist in a crisis situation, and/or implement restraint. As a result, they were unfamiliar with comprehensive restraint protocols. This finding is consistent with a recent literature review indicating that the rapid response teams commonly used to assist with acute medical crises have more recently been adapted to help manage psychiatric crises (Choi et al., 2019). Behavioral rapid response teams (BRRTs) are led by a nurse and security guard at minimum, and often do not include physicians. Once a response team is alerted, medical trainees and physicians are expected to attend to other patients; one medical trainee participant reported receiving guidance from an attending physician to continue treating other patients while the severe behavior was managed by the BRRT.

Although studies demonstrate that BRRTs help improve medical outcomes for the general patient population (e.g., cardiac arrest; Bellomo et al., 2003) and behavioral outcomes for patients with psychiatric crisis (e.g., reduced restraint use; Choi et al., 2019), there is little research about the use of BRRTs to manage patients with ASD and severe behavior. Given the demands placed on physicians and the wide range of clients they treat, relying on BRRTs may be an effective means to assist with managing severe behavior in patients with ASD. However, this does not alleviate the need for hospitals and/or medical schools to provide better training on managing severe behavior in patients with ASD. Teams called in to manage these patients' severe behavior must possess practical skills for assessing behavioral function and safely treating neurodiverse patients. Future research should investigate the competencies and ASDspecific training provided to BRRT members.

Meanwhile, study participants uniformly indicated that they wished they had more formal medical training specific to treating patients with ASD. The results of the ASK-Q indicated appropriate overall knowledge of ASD among the participants; however, being able to identify or describe ASD's core features does not translate into practical knowledge about addressing these features in a hospital setting. Moreover, several medical trainees' ASK-Q sub-scale scores indicated stigma associated with ASD, suggesting a need for specialized training that can not only help to improve the standard of hospital care for patients with ASD across the lifespan but also decrease potential stigma associated with treating this population. A recent study found that patients and caregivers are likely to report a positive medical experience when physicians have ASD-specific knowledge, provide detailed explanations of the exam or procedure, and use positive reinforcement (Wilson \& Peterson, 2018). Given the increasing prevalence of ASD nationwide (Christensen et al., 2019) and the many challenging features of hospital environments for patients with ASD, training specific to treating patients with ASD and severe behavior is likely to become even more important in future years.

One participant suggested including simulated patients with ASD in medical school training. Simulated patients, trained actors who portray a predetermined set of symptoms or a specific diagnosis (Kaplonyi et al., 2017; Williams \& Song, 2016), are effective in allowing medical trainees to practice and refine technical, non-technical clinical (e.g., communication), and cognitive skills (Kaplonyi et al., 2017; Williams \& Song, 2016). A standardized simulated patient that accurately portrays the core features of ASD may increase both physician competency and comfort in treating this unique patient population. Strategic exposure to different features of ASD that may manifest during hospital visits has the potential to prepare physicians to quickly adapt their approach to support patients exhibiting severe behavior.

Interestingly, although restraint is typically implemented by teams that do not include physicians, participants reported that the attending physician is responsible for documenting the justification for restraint, restraint type, and follow-up assessment. They also noted that nurses often need to remind physicians to complete assessment procedures and subsequent documentation. Moreover, participants explained that restraint documentation may not include data that would be helpful to 
others who treat the patient later such as restraint duration; patient's, caregivers', and surrounding patients' responses to restraint; and if/when/how a post-restraint explanation was provided. In fact, participants identified some limitations to their hospital's electronic health records system which make it difficult for physicians to find relevant information about patients with ASD who have a history of severe behavior. They reported that the flagging system designed to alert the physician to a particular presenting concern or diagnosis and the 'problem list' section are not used consistently. Participants noted that flagging a patient's record currently requires special paperwork and processing through institutional departments, which seems unnecessarily time consuming, and they pointed out that unified smartphrases (i.e., note templates) could facilitate efficient, consistent documentation of behavioral information in problem lists. They further noted that if the documentation process were standardized, physicians could more easily focus on relevant pieces of documentation within a patient's electronic file. In addition to the potential of ACPs to improve the quality of healthcare services for patients with ASD and their families (Broder-Fingert et al., 2016), these plans could facilitate streamlined electronic health record documentation systems. For example, ACPs could be expanded to include detailed behavioral information about a patient and integrated with alerts to physicians to review the plan prior to treating a patient.

Whereas others have reported using chemical restraint to reactively manage severe behavior in adults (Friedman \& Crabb, 2018), participants in this study indicated that chemical restraint was most often applied to proactively manage characteristics of ASD or to facilitate completion of medical procedures. This finding is consistent with proactive use of chemical restraint to facilitate safe medical procedures in pediatric patients (Kirwan \& Coyne, 2017). Differences in the literature could be due to differing interpretations of the term "chemical restraint"; study participants may characterize reactive medication administration as "medicating emergent behaviors."

Although this study produced several important findings, there are several limitations to note. Given that the primary aim of this research was to gather physician experiences treating patients with ASD and severe behavior, obtaining knowledge of ASD was a secondary goal and we did not want to cause physicians to question their expertise prior to participating. However, completion of the ASK-Q questionnaire immediately following focus group participation may have resulted in an increase in knowledge of ASD and decreased stigma from discussing these topics with their colleagues. Further, given participant-reported concerns with documentation of diagnosis and accessing of information in electronic health records, it is possible that physicians may be unaware of an ASD diagnosis in some patients treated for unrelated medical needs, thus influencing their comprehensive reporting of all patients treated with ASD. In addition, restraint can be a controversial, uncomfortable topic for physicians, which may have impacted focus group and/or interview responses. Participants may have hesitated to elaborate on open-ended questions due to a desire to present as competent and using evidence-based clinical practice (e.g., not endorsing overuse of restraint). To reduce potential discomfort, participants were encouraged to report not only their experiences but also observed experiences of others, so they did not need to take ownership of treatment decisions that might be negatively perceived. To facilitate open discussion among medical trainees including identifying potential improvements in their work environments, focus groups were limited to trainees. With all qualitative research, there is the potential that facilitators misjudged session saturation which may have led to premature conclusion of data collection and, thus, inadequate sample sizes (Braun \& Clarke, 2006). Instead, researchers have moved past theoretical saturation to consider pragmatic saturation (rather than being an absolute end point; Low, 2019) and emphasized the concept of information power (i.e., the greater the relevant information in the sample, the fewer participants needed; Malterud et al., 2016), which both suggest that qualitative researchers use interpretative judgement related to the study aims to determine when the number of participants is sufficient. The total sample size in the current study is adequate relative to the purposes of this study, as supported by the literature (Malterud et al., 2016; Marshall et al., 2013; Sim et al., 2018). However, subgroup composition may be a limitation of this study given that a majority of participants were medical trainees, specifically medical students. Recruiting early-career physicians proved to be difficult, possibly due to additional stressors placed on them during the COVID-19 pandemic, which may have decreased their availability and/or willingness to participate in research outside of scheduled patient hours.

However, the three early-career physicians who did participate in this study echoed the same themes as medical trainees. Moreover, medical trainees are known to be a valuable population to target when aiming to improve quality of medical research and practice (Gould et al., 2002) and they are generally considered to be representative of the larger medical population. Future investigations should also include physicians with more experience as more clinical exposure might increase practical knowledge and skills related to the treatment of severe behavior in patients with ASD.

This exploratory study represents a valuable first step towards improving healthcare for patients with ASD. Results from focus groups and interviews discussing physicians' experiences treating patients with ASD and severe behavior at an urban teaching hospital in the northeastern United States indicate a need for more hospital staff training in how to manage severe behavior in patients with ASD. Future research should explore how to most efficiently and 
effectively train hospital staff in managing severe behavior in patients with ASD. This work also highlights the need for research focused on establishing consistent hospital protocols for supporting patients with ASD and severe behavior, which should include developing best practices for documentation such as consistent use of flags and a specific vocabulary to be used in problem lists and progress notes. Finally, future research in this area should drive adaptations to electronic health records which facilitate consistent use of flags and standardized language to describe behaviors and behavior management for hospital patients with ASD.

\section{Appendix 1}

Focus group question guide.

\section{Topic 1: Autism}

1. Describe your experience treating individuals with autism i. Prompt: Across the lifespan

2. What training or education have you received specific to autism?

3 . How would you approach treating a patient with autism vs. a patient without autism?

Topic 2: Severe Behavior

4. What do you consider to be severe behavior?

5. Describe your experience(s) with patients with autism who engage in severe behavior in a hospital setting

i. Prompt: Across the lifespan

6. Describe how you would manage severe behavior in patients with autism vs. those without autism

7. Why do you think that patients with autism engage in severe behavior?

8. What do you consider a crisis situation?

9. If you had a patient in your department with autism who aggressed toward you, what would you do?

10. If you had a patient in your department with autism who was engaging in severe head banging against a hard surface, what would you do?

Topic 3: Restraint Implementation

11. Describe specific instances in which restraint was used in your department

i. Prompt: Behavior description, restraint implementer, restraint type

12. Describe crisis and restraint training you have received throughout the course of your professional career

13. Describe any variables that impact restraint use

Topic 4: Post-restraint

14. Describe how your patients and/or families have reacted to restraint use

15. Describe the protocols you have to follow after restraint use 16. Do you know ahead of time if your patient has a history of severe behavior?

17. What do you think would help you to better treat patients with autism who engage in severe behavior?

Closing

18. Is there anything else you would like to share about restraint or severe behavior in patients with autism?
Author contributions All authors contributed to the study conceptualization and design. Material preparation, data collection and analysis were performed by Giovanna Salvatore. Funding acquisition was completed by Giovanna Salvatore and Christina Simmons. The first draft of the manuscript was written by Giovanna Salvatore and all authors commented on previous versions of the manuscript. All authors read and approved the final manuscript.

Funding This manuscript is in partial fulfillment of first author's Master's degree. Funding for this study was provided by a Graduate Research Grant from the Organization for Autism Research and a Camden Health Research Initiative Grant from Rowan University.

\section{References}

Allen, D., Lowe, K., Brophy, S., \& Moore, K. (2009). Predictors of restrictive reactive strategy use in people with challenging behaviour. Journal of Applied Research in Intellectual Disabilities, 22(2), 159-168. https://doi.org/10.1111/j.1468-3148.2008. 00484.X

American Psychiatric Association. (2013). Diagnostic and statistical manual of mental disorders: Diagnostic and statistical manual of mental disorders (5th ed.). American Psychiatric Association.

Association of American Medical Colleges. (n.d.). Diversity in Medicine: Facts and figures 2019. Retrieved from https://www.aamc. org/data-reports/workforce/report/diversity-medicine-facts-andfigures-20199.

Bellomo, R., Goldsmith, D., Uchino, S., Buckmaster, J., Hart, G. K., Opdam, H., Silvester, W., Doolan, L., \& Gutteridge, G. (2003). A prospective before-and-after trial of a medical emergency team. Medical Journal of Australia, 179(6), 283-287. https://doi.org/ 10.5694/j.1326-5377.2003.tb05548.x

Bender, D. E., \& Ewbank, D. (1994). The focus group as a tool for health research: Issues in design and analysis. Health Transition Review, 4(1), 63-80.

Blumberg, G. K., \& Roppolo, L. P. (2021). Restraint and Seclusion in the Emergency Department. In L. S. Zun, K. Nordstrom, \& M. P. Wilson (Eds.), Behavioral emergencies for healthcare providers (pp. 249-256). Springer. https://doi.org/10.1007/9783-030-52520-0_24

Braun, V., \& Clarke, V. (2006). Using thematic analysis in psychology. Qualitative Research in Psychology, 3(2), 77-101. https:// doi.org/10.1191/1478088706qp063oa

Broder-Fingert, S., Shui, A., Ferrone, C., Iannuzzi, D., Cheng, E. R., Giauque, A., Connors, S., McDougle, C. J., Donelan, K., Neumeyer, A., \& Kuhlthau, K. (2016). A pilot study of autism-specific care plans during hospital admission. Pediatrics, 137(Suppl. 2), S196-S204. https://doi.org/10.1542/peds. 2015-2851R

Buescher, A. V., Cidav, Z., Knapp, M., \& Mandell, D. S. (2014). Costs of autism spectrum disorders in the United Kingdom and the United States. Journal of the American Medical Association Pediatrics, 168(8), 721-728. https://doi.org/10.1001/jamapediat rics. 2014.210

Campbell, J. M. (2003). Efficacy of behavioral interventions for reducing problem behavior in persons with autism: A quantitative synthesis of single-subject research. Research in Developmental Disabilities, 24(2), 120-138. https://doi.org/10.1016/S0891-4222(03) 00014-3 
Choi, K. R., Omery, A. K., \& Watkins, A. M. (2019). An integrative literature review of psychiatric rapid response teams and their implementation for de-escalating behavioral crises in nonpsychiatric hospital settings. The Journal of Nursing Administration, 49(6), 297-302. https://doi.org/10.1097/NNA.0000000000000756

Christensen, D. L., Maenner, M. J., Bilder, D., Constantino, J. N., Daniels, J., Durkin, M. S., Fitzgerald, R. T., Kurzius-Spencer, M., Pettygrove, S. D., Robinson, C., Shenouda, J., White, T., Zahorodny, W., Pazol, K., \& Dietz, P. (2019). Prevalence and characteristics of autism spectrum disorder among children aged 4 years-Early autism and developmental disabilities monitoring network, seven sites, United States, 2010, 2012, and 2014. Morbidity and Mortality Weekly Report Surveillance Summaries, 68(2), 1-19. https:// doi.org/10.15585/mmwr.ss6802a1

Cosper, P., Morelock, V., \& Provine, B. (2015). Please release me: Restraint reduction initiative in a health care system. Journal of Nursing Care Quality, 30(1), 16-23. https://doi.org/10.1097/NCQ. 0000000000000074

Day, R. M., Rea, J. A., Schussler, N. G., Larsen, S. E., \& Johnson, W. L. (1988). A functionally based approach to the treatment of self-injurious behavior. Behavior Modification, 12(4), 565-589. https://doi.org/10.1177/01454455880124005

Deavenport-Saman, A., Lu, Y., Smith, K., \& Yin, L. (2016). Do children with autism overutilize the emergency department? Examining visit urgency and subsequent hospital admissions. Maternal and Child Health Journal, 20(2), 306-314. https://doi.org/10. 1007/s10995-015-1830-y

Delaney, K. R., \& Fogg, L. (2005). Patient characteristics and setting variables related to use of restraint on four inpatient psychiatric units for youths. Psychiatric Services, 56(2), 186-192. https://doi. org/10.1176/appi.ps.56.2.186

Emerson, E., Kiernan, C., Alborz, A., Reeves, D., Mason, H., Swarbrick, R., Mason, L., \& Hatton, C. (2001). The prevalence of challenging behaviors: A total population study. Research in Developmental Disabilities, 22(1), 77-93. https://doi.org/10.1016/ S0891-4222(00)00061-5

Evans, L. K., \& Cotter, V. T. (2008). Avoiding restraints in patients with dementia: Understanding, prevention, and management are the keys. American Journal of Nursing, 108(3), 40-49. https://doi. org/10.1097/01.NAJ.0000311827.75816.8b

Friedman, C., \& Crabb, C. (2018). Restraint, restrictive intervention, and seclusion of people with intellectual and developmental disabilities. Intellectual and Developmental Disabilities, 56(3), 171-187. https://doi.org/10.1352/1934-9556-56.3.171

Gabriels, R. L., Agnew, J. A., Beresford, C., Morrow, M. A., Mesibov, G., \& Wamboldt, M. (2012). Improving psychiatric hospital care for pediatric patients with autism spectrum disorders and intellectual disabilities. Autism Research and Treatment. https://doi. org/10.1155/2012/685053

Glaser, B. G., \& Strauss, A. (1967). The discovery of grounded theory: Strategies for qualitative research. Aldine.

Golnik, A., Ireland, M., \& Borowsky, I. W. (2009). Medical homes for children with autism: A physician survey. Pediatrics, 123(3), 966-971. https://doi.org/10.1542/peds.2008-1321

Gould, B. E., Grey, M. R., Huntington, C. G., Gruman, C., Rosen, J. H., Storey, E., Abrahamson, L., Conaty, A. M., Curry, L., Ferrerira, M., Harrington, K. L., Paturzo, D., \& Van Hoof, T. J. (2002). Improving patient care outcomes by teaching quality improvement to medical students in community-based practices. Academic Medicine, 77(10), 1011-1018. https://doi.org/10.1097/00001888200210000-00014

Grimes, R. D. (2012). Predicting involvement in coercive interventions from individual and contextual risk factors and treatment context [Doctoral thesis, The University of Alabama], The University of Alabama Institutional Repository.
Hagopian, L. P., Bruzek, J. L., Bowman, L. G., \& Jennett, H. K. (2007). Assessment and treatment of problem behavior occasioned by interruption of free-operant behavior. Journal of Applied Behavior Analysis, 40(1), 89-103. https://doi.org/10.1901/jaba.2007.63-05

Harrison, A. J., Bradshaw, L. P., Naqvi, N. C., Paff, M. L., \& Campbell, J. M. (2017). Development and psychometric evaluation of the autism stigma and knowledge questionnaire (ASK-Q). Journal of Autism and Developmental Disorders, 47(10), 3281-3295. https:// doi.org/10.1007/s10803-017-3242-x

Harrison, A. J., Paff, M. L., \& Kaff, M. S. (2019). Examining the psychometric properties of the autism stigma and knowledge questionnaire (ASK-Q) in multiple contexts. Research in Autism Spectrum Disorders, 57, 28-34. https://doi.org/10.1016/j.rasd. 2018.10.002

Hazen, E. P., Ravichandran, C., Hureau, A. R., O'Rourke, J., Madva, E., \& McDougle, C. J. (2020). Agitation in patients with autism spectrum disorder admitted to inpatient pediatric medical units. Pediatrics, 145(Suppl. 1), S108-S116. https://doi.org/10.1542/ peds.2019-1895N

Heyvaert, M., Saenen, L., Campbell, J. M., Maes, B., \& Onghena, P. (2014). Efficacy of behavioral interventions for reducing problem behavior in persons with autism: An updated quantitative synthesis of single-subject research. Research in Developmental Disabilities, 35(10), 2463-2476. https://doi.org/10.1016/j.ridd. 2014.06.017

Hill, A. P., Zuckerman, K. E., Hagen, A. D., Kriz, D. J., Duvall, S. W., Van Santen, J., Nigg, J., Fair, D., \& Fombonne, E. (2014). Aggressive behavior problems in children with autism spectrum disorders: Prevalence and correlates in a large clinical sample. Research in Autism Spectrum Disorders, 8(9), 1121-1133. https:// doi.org/10.1016/j.rasd.2014.05.006

Iwata, B. A., Dorsey, M. F., Slifer, K. J., Bauman, K. E., \& Richman, G. S. (1982/1994). Toward a functional analysis of self-injury. Journal of Applied Behavior Analysis, 27, 197-209 (Reprinted from Analysis and Intervention in Developmental Disabilities, 2, 3-20 1982). https://doi.org/10.1901/jaba.1994.27-197

Johnson, N. L., \& Rodriguez, D. (2013). Children with autism spectrum disorder at a pediatric hospital: A systematic review of the literature. Pediatric Nursing, 39(3), 131-141.

Kanne, S. M., \& Mazurek, M. O. (2011). Aggression in children and adolescents with ASD: Prevalence and risk factors. Journal of Autism and Developmental Disorders, 41(7), 926-937. https:// doi.org/10.1007/s10803-010-1118-4

Kaplonyi, J., Bowles, K. A., Nestel, D., Kiegaldie, D., Maloney, S., Haines, T., \& Williams, C. (2017). Understanding the impact of simulated patients on health care learners' communication skills: A systematic review. Medical Education, 51(12), 1209-1219. https://doi.org/10.1111/medu.13387

Kirwan, L., \& Coyne, I. (2017). Use of restraint with hospitalized children: A survey of nurses' perceptions of practices. Journal of Child Health Care, 21(1), 46-54. https://doi.org/10.1177/13674 93516666730

Kupzyk, S., \& Allen, K. D. (2019). A review of strategies to increase comfort and compliance with medical/dental routines in persons with intellectual and developmental disabilities. Journal of Developmental and Physical Disabilities, 31(2), 231-249. https://doi. org/10.1007/s10882-018-09656-y

Larue, C., Dumais, A., Ahern, E., Bernheim, E., \& Mailhot, M. P. (2009). Factors influencing decisions on seclusion and restraint. Journal of Psychiatric and Mental Health Nursing, 16(5), 440446. https://doi.org/10.1111/j.1365-2850.2009.01396.x

Liu, G., Pearl, A. M., Kong, L., Leslie, D. L., \& Murray, M. J. (2017). A profile on emergency department utilization in adolescents and young adults with autism spectrum disorders. Journal of Autism and Developmental Disorders, 47(2), 347-358. https://doi.org/10. 1007/s10803-016-2953-8 
Lokhandwala, T., Khanna, R., \& West-Strum, D. (2012). Hospitalization burden among individuals with autism. Journal of Autism and Developmental Disorders, 42(1), 95-104. https://doi.org/10. 1007/s10803-011-1217-x

Low, J. (2019). A pragmatic definition of the concept of theoretical saturation. Sociological Focus, 52(2), 131-139. https://doi.org/ 10.1080/00380237.2018.1544514

Lunsky, Y., Paquette-Smith, M., Weiss, J. A., \& Lee, J. (2014). Predictors of emergency service use in adolescents and adults with autism spectrum disorder living with family. Emergency Medicine Journal, 32(10), 787-792. https://doi.org/10.1136/emerm ed-2014-204015

Malterud, K., Siersma, V. K., \& Guassora, A. D. (2016). Sample size in qualitative interview studies: Guided by information power. Qualitative Health Research, 26(13), 1753-1760. https://doi.org/ 10.1177/1049732315617444

Mann-Poll, P. S., Smit, A., de Vries, W. J., Boumans, C. E., \& Hutschemaekers, G. J. (2011). Factors contributing to mental health professionals' decision to use seclusion. Psychiatric Services, 62(5), 498-503. https://doi.org/10.1176/ps.62.5.pss6205_0498

Marshall, B., Cardon, P., Poddar, A., \& Fontenot, R. (2013). Does sample size matter in qualitative research?: A review of qualitative interviews in IS research. Journal of Computer InformationSsystems, 54(1), 11-22. https://doi.org/10.1080/08874417.2013. 11645667

Mazurek, M. O., Harkins, C., Menezes, M., Chan, J., Parker, R. A., Kuhlthau, K., \& Sohl, K. (2020). Primary care providers' perceived barriers and needs for support in caring for children with autism. Journal of Pediatrics, 221, 240-245. https://doi.org/10. 1016/j.jpeds.2020.01.014

McTiernan, A., Leader, G., Healy, O., \& Mannion, A. (2011). Analysis of risk factors and early predictors of challenging behavior for children with autism spectrum disorder. Research in Autism Spectrum Disorders, 5(3), 1215-1222. https://doi.org/10.1016/j. rasd.2011.01.009

Morgan, D. L. (1997). Focus groups as qualitative research (2nd ed.). SAGE Publications.

Murphy, O., Healy, O., \& Leader, G. (2009). Risk factors for challenging behaviors among 157 children with autism spectrum disorder in Ireland. Research in Autism Spectrum Disorders, 3(2), 474-482. https://doi.org/10.1016/j.rasd.2008.09.008

Muskat, B., Burnham Riosa, P., Nicholas, D. B., Roberts, W., Stoddart, K. P., \& Zwaigenbaum, L. (2015). Autism comes to the hospital: The experiences of patients with autism spectrum disorder, their parents and health-care providers at two Canadian paediatric hospitals. Autism, 19(4), 482-490. https://doi.org/10.1177/13623 61314531341

Newcomb, E. T., \& Hagopian, L. P. (2018). Treatment of severe problem behaviour in children with autism spectrum disorder and intellectual disabilities. International Review of Psychiatry, 30(1), 96-109. https://doi.org/10.1080/09540261.2018.1435513

O’Donoghue, E. M., Pogge, D. L., \& Harvey, P. D. (2020). The impact of intellectual disability and autism spectrum disorder on restraint and seclusion in pre-adolescent psychiatric inpatients. Journal of Mental Health Research in Intellectual Disabilities, 13(2), 86-109. https://doi.org/10.1080/19315864.2020.1750742

Oskoui, M., \& Wolfson, C. (2012). Treatment comfort of adult neurologists in childhood onset conditions. Canadian Journal of Neurological Sciences, 39(2), 202-205. https://doi.org/10.1017/S0317 167100013238

Riley, A. R., \& Freeman, K. A. (2019). Impacting pediatric primary care: Opportunities and challenges for behavioral research in a shifting healthcare landscape. Behavior Analysis: Research and Practice, 19(1), 23-38. https://doi.org/10.1037/bar0000114
Roy, C., Castonguay, A., Fortin, M., Drolet, C., Franche-Choquette, G., Dumais, A., \& Geoffrion, S. (2019). The use of restraint and seclusion in residential treatment care for youth: A systematic review of related factors and interventions. Trauma, Violence, \& Abuse, 22(2), 318-338. https://doi.org/10.1177/1524838019 843196

Schnitzer, K., Merideth, F., Macias-Konstantopoulos, W., Hayden, D., Shtasel, D., \& Bird, S. (2020). Disparities in care: The role of race on the utilization of physical restraints in the emergency setting. Academic Emergency Medicine, 27(10), 943-950. https://doi.org/ 10.1111/acem.14092

Sim, J., Saunders, B., Waterfield, J., \& Kingstone, T. (2018). Can sample size in qualitative research be determined a priori? International Journal of Social Research Methodology, 21(5), 619-634. https://doi.org/10.1080/13645579.2018.1454643

Stewart, D. W., \& Shamdasani, P. (2017). Online focus groups. Journal of Advertising, 46(1), 48-60. https://doi.org/10.1080/00913367. 2016.1252288

Strauss, A. L. (1987). Qualitative analysis for social scientists. Cambridge University Press.

Sturmey, P. (2018). Reducing restraint in individuals with intellectual disabilities and autism spectrum disorders: A systematic review group interventions. Advances in Neurodevelopmental Disorders, 2(4), 375-390. https://doi.org/10.1007/s41252-018-0088-y

van Steensel, F. J., Bögels, S. M., \& Perrin, S. (2011). Anxiety disorders in children and adolescents with autistic spectrum disorders: A meta-analysis. Clinical Child and Family Psychology Review, 14(3), 302-317. https://doi.org/10.1007/s10567-011-0097-0

Werner, S., \& Shulman, C. (2013). Subjective well-being among family caregivers of individuals with developmental disabilities: The role of affiliate stigma and psychosocial moderating variables. Research in Developmental Disabilities, 34(11), 4103-4114. https://doi.org/10.1016/j.ridd.2013.08.029

Williams, B., \& Song, J. J. Y. (2016). Are simulated patients effective in facilitating development of clinical competence for healthcare students? A Scoping Review. Advances in Simulation, 1(1), 1-9. https://doi.org/10.1186/s41077-016-0006-1

Wilson, S. A., \& Peterson, C. C. (2018). Medical care experiences of children with autism and their parents: A scoping review. Child: Care, Health and Development, 44(6), 807-817. https://doi.org/ 10.1111/cch.12611

Wong, A. H., Ray, J. M., Rosenberg, A., Crispino, L., Parker, J., McVaney, C., Iennaco, J. D., Bernstein, S. L., \& Pavlo, A. J. (2020). Experiences of individuals who were physically restrained in the emergency department. Journal of the American Medical Association Network Open, 3(1), e1919381-e1919381. https://doi. org/10.1001/jamanetworkopen.2019.19381

Wong, A. H., Taylor, R. A., Ray, J. M., \& Bernstein, S. L. (2019). Physical restraint use in adult patients presenting to a general emergency department. Annals of Emergency Medicine, 73(2), 183-192. https://doi.org/10.1016/j.annemergmed.2018.06.020

Zerbo, O., Massolo, M. L., Qian, Y., \& Croen, L. A. (2015). A study of physician knowledge and experience with autism in adults in a large integrated healthcare system. Journal of Autism and Developmental Disorders, 45(12), 4002-4014. https://doi.org/10.1007/ s10803-015-2579-2

Publisher's Note Springer Nature remains neutral with regard to jurisdictional claims in published maps and institutional affiliations. 